MAD-TH-08-01

\title{
A Note on Spontaneously Broken Lorentz Invariance
}

\author{
Akikazu Hashimoto \\ Department of Physics, University of Wisconsin, Madison, WI 53706
}

\begin{abstract}
We consider a relativistic effective field theory of vector boson whose vacuum exhibits spontaneous breaking of Lorentz invariance. We argue that a simple model of this type, considered recently by Kraus and Tomboulis, is obstructed from having a consistent ultraviolet completion according to the diagnostic recently suggested by Adams, Arkani-Hamed, Dubovsky, Nicolis, and Rattazzi.
\end{abstract}


Relativistic quantum field theory, formally defined as Lorentz invariant ultraviolet (UV) fixed point and a subsequent renormalization group flow, is an elegant formalism successfully capturing the physics of elementary particles. As a quantum theory, they rest on the foundation of unitary evolution of states in the Hilbert space. States themselves form a representation of the Lorentz group, and the dynamics dictates the spectrum of states and their interactions.

Gauge principle is a critical ingredient for keeping unitarity and Lorentz invariance mutually compatible in models including states of spin one (or higher). When combined with the requirement that the UV fixed point is a weakly coupled field theory with a Lagrangian formulation, the list of possible models is incredibly short. They essentially consist solely of asymptotically free gauge theories minimally coupled to matter fields which are spin zero or spin half. It is therefore quite remarkable that QCD is, and that the Standard Model admits a UV completion of 1 a model of this type.

A closely related and familiar formalism in a theorist's toolbox is the effective field theory (reviewed, e.g., in [2, 3, 4]). Roughly speaking, effective field theory is the result of flowing down the renormalization group from the UV to some scale of interest from the point of view of a probe or a physical process. It is not possible to track the flow of all the parameters of the renormalization group in closed analytic form. Nonetheless, one can draw useful conclusions about the strength of physical effects through systematic analysis of the dimensions of operators in the effective action, energy scales, and symmetries, within the framework of effective field theory. A productive and frequently adopted attitude in the use of effective field theory technique is to not dwell on the UV completion. By following this dictum, model builders in cosmology, astrophysics, and particle physics are free to introduce wide range of exotic models and to study their implications.

In light of the restrictiveness imposed by the consistency, however, there always persist some degree of doubt that a given effective field theory model might not admit a consistent UV completion. This is especially the case for exotic models exhibiting features such as the spontaneous breaking of Lorentz invariance. It is not difficult to imagine that effective field theories arising from a consistent UV complete theories are somehow restricted, e.g., in the numerical values of the coefficients of various operators in the effective action. These conditions have not been explored systematically simply because they are difficult to derive from first principles.

Along this line of thought, a simple connection relating positivity of forward scattering amplitude, possibility of superluminal propagation in a non-trivial background, and a sign of certain irrelevant operators in the effective field theory, was pointed out recently by Adams,

\footnotetext{
${ }^{1}$ An example of embedding of the Standard Model in a conformal UV fixed point was described in [1].
} 
et.al. in [5]. A prototype of their argument is an effective field theory of the form

$$
L=\int d^{4} x\left[\frac{1}{2} \partial_{\mu} \phi \partial^{\mu} \phi+c\left(\partial_{\mu} \phi \partial^{\mu} \phi\right)^{2}\right]
$$

We take the space-time to be Minkowski. Since much of the discussion boils down to that of a sign, let us also specify that we are using the metric signature convention $(+,-,-,-)$. The authors of [5] have argued that the sign of $c$ must be positive if such an effective field theory is to arise from a consistent UV complete theory, by showing that theories with negative values of $c$ exhibits pathologies which manifests itself in the form of a superluminal propagation in a non-trivial background. The authors of [5] also showed that the negative sign of $c$ is in conflict with analyticity of the forward scattering amplitudes. These findings further highlight the significance of the sign of $c$ as a way to diagnose whether a certain effective field theory can arise from a relativistic UV fixed point theory. The authors of [5] identified the induced gravity models of Dvali, Gabadadze, and Porrati [6] as an example of a model which appears not to admit a UV completion from this point of view.

In this article, we investigate the status of a model of spontaneous Lorentz symmetry breaking along similar lines. More specifically, we study a model whose effective Lagrangian (so the theory has a cut-off) has the form 2

$$
L=\int d^{4} x N\left[-\frac{1}{4} F_{\mu \nu} F^{\mu \nu}-V\left(A_{\mu} A^{\mu}\right)\right] .
$$

The parameter $N$ is a dimensionless constant and is taken to be large so that the theory is weakly coupled. The vector field interacts via the Lorentz invariant interaction term $V\left(A^{2}\right)$, which one might take to be of the form

$$
V\left(A^{2}\right)=\frac{\lambda}{4}\left(A_{\mu} A^{\mu}-v\right)^{2}
$$

which is minimized at

$$
A^{2}=A_{\mu} A^{\mu}=v .
$$

This can a priori can be either positive (time-like) or negative (space-like) depending on the sign of $v$. More generally, we take $V\left(A^{2}\right)$ to admit a power series expansion in $A^{2}$ of the form

$$
V\left(A^{2}\right)=v_{2} A^{2}+v_{4}\left(A^{2}\right)^{2}+v_{6}\left(A^{2}\right)^{3}+\ldots, \quad v_{n} \sim \Lambda^{4-n} .
$$

The goal of this note is to argue that (2) is an inconsistent effective field theory according to the diagnostic criteria of [5].

\footnotetext{
${ }^{2}$ Effective field theory models of spontaneous breaking of Lorentz invariance have been studied extensively by Kostelecky and collaborators, e.g., in [7,8,9].
} 
The general idea that photons might arise as a Goldstone boson for spontaneously broken Lorentz invariance has a long history and appears to go back to Bjorken [10] who considered a fermion model similar to that of Nambu and Jona-Lasinio [1] but with a condensation in the vector component of the fermion biliniear

$$
L=\bar{\psi}_{i}(i \not \partial-m) \psi_{i}+\lambda_{2}\left(\bar{\psi}_{i} \gamma^{\mu} \psi_{i}\right)^{2}
$$

The original vision of Bjorken was to obtain a Lorentz invariant dynamics of quantum electrodynamics in a non-Lorentz invariant gauge. It was later pointed out by Banks and Zaks [12], however, that the violation of gauge invariance is physical and has an effect on gauge invariant observables. More recently, Kraus and Tomboulis studied an effective field theory precisely of the form (2) and argued that

- such an action can arise as an effective dynamics for a model similar to that of Bjorken where $N$ corresponds to the number of fermion fields 3 and

- that the observable effects of Lorentz invariance can be made parametrically small by taking $N$ to be large.

To relate (2) to models of fermion bilinear condensates, Kraus and Tomboulis considered a model of the form

$$
L=\bar{\psi}_{i}(i \not \partial-m) \psi_{i}+N \sum_{n=1}^{\infty} \lambda_{2 n} \frac{\left(\bar{\psi}_{i} \gamma^{\mu} \psi_{i}\right)^{2 n}}{N^{2 n}}
$$

with the dimensionless coupling $\lambda_{2 n} / \Lambda^{4-6 n}$ being a number of order one. An effective field theory of this form might arise, for example, by integrating out a massive vector boson of mass $\Lambda$ with respect to which the fermions are charged.

The action (7) can be re-written using the standard trick of introducing an auxiliary field $A^{\mu}$

$$
L=\bar{\psi}_{i}(i \not \partial-\not A-m) \psi_{i}-N V\left(A_{\mu} A^{\mu}\right)
$$

In this formulation, $A_{\mu}$ is non-dynamical and imposes a constraint

$$
\bar{\psi}_{i} \gamma_{\mu} \psi_{i}+2 N A_{\mu} V^{\prime}\left(A^{2}\right)=0
$$

and when $A_{\mu}$ is eliminated, reduces (8) to (77) where

$$
\lambda_{2}=\frac{1}{4 v_{2}}, \quad \lambda_{4}=-\frac{v_{4}}{16 v_{2}^{4}}, \quad \lambda_{6}=\frac{4 v_{4}^{2}-v_{2} v_{6}}{64 v_{2}^{7}}, \quad \ldots .
$$

\footnotetext{
${ }^{3}$ The connection between the fermion model and the effective vector model was analyzed critically in [13]. We thank Per Kraus for bringing this article to our attention. Independent of this observation, we can explore the UV completability issue for the effective field theory of vector boson model (17).
} 
If instead we integrate the fermions out first, a gauge invariant kinetic term of order $N$ is induced and we recover an effective action of the form (2), up to numerical factors of order one and additional higher derivative operators.

The case where only $v_{2}$ and $\lambda_{2}$ are non-vanishing corresponds to the model of Bjorken. The effective action in terms of $A_{\mu}$ field is the Proca action

$$
L=\int d^{4} x N\left[-\frac{1}{4} F_{\mu \nu} F^{\mu \nu}+\frac{\mu^{2}}{2} A_{\mu} A^{\mu}\right]
$$

where

$$
\mu^{2}=-2 v_{2}=-\frac{1}{2 \lambda_{2}}
$$

The resulting dynamics, however, is crucially sensitive to the sign of $\lambda_{2}$ [10, 12]. If $\lambda_{2}$ is negative, space-like components of the $A_{\mu}$ are stable and we expect to find the usual effective dynamics of massive vector bosons. This theory is in fact renormalizable and BRST invariant, and can be quantized perturbatively when coupled minimally to matter [14]. If $\lambda_{2}$ is positive, one expects a run away behavior, which must somehow be stabilized in order for the theory to have a vacuum. Higher order operators in $V\left(A^{\mu} A_{\mu}\right)$ were introduced specifically for this purpose [15, 16].

While the effective Lagrangian (2) does appear to arise from a model of interacting fermions (7), it does not necessarily follow that (2) can be embedded into a consistent UV fixed point theory. The reason is simply the fact that (7) is not a renormalizable field theory. While an effective action whose general from is that of (7) could arise from integrating out a massive vector field, it is not obvious that there are enough freedom to control the values of the coupling constants $\lambda_{2 n}$ in the effective action. It is unnatural, in particular, for $\lambda_{2}$ to take on a positive value as a result of integrating out a massive vector boson, which corresponds to fermion/anti-fermion interactions being repulsive.

More can be said about the range of possible values of $\lambda_{2 n}$ if we were to consider this model in $1+1$ dimensions. There, we can take advantage of bosonisation techniques (reviewed e.g. in [17, 18, 19]) to study these models in greater detail. The $1+1$ dimensional version of (7) is in fact a generalization of the Thirring model which can be analyzed along the lines of [20,21,22], with additional higher order interactions of the form

$$
\lambda_{2 n}\left(J_{\mu} J^{\mu}\right)^{n} \sim \lambda_{2 n}\left(\left(\bar{\psi} \gamma_{\mu} \psi\right)\left(\bar{\psi} \gamma^{\mu} \psi\right)\right)^{n} \sim \lambda_{2 n}\left(\partial_{\mu} \phi \partial^{\mu} \phi\right)^{n}
$$

In terms of the bosonized scalar field, this model resembles the "Ghost condensation" model 23] if $\lambda_{2}$ is positive, and might also contain an additional sine-Gordon potential if the fermions are massive. The coupling constant $\lambda_{4}$ is naturally seen in this formulation to be constrained by the diagnostic of [5]. It is very natural to expect the values $\lambda_{2 n}$ of the irrelevant 
fermion couplings in $3+1$ dimensions to be constrained along similar lines, although we were unable to find a simple formulation of it at the present time.

These considerations, however, more directly concerns the fermion model (7) than the effective theory (2). In fact, it is well known that in $1+1$ dimensions, the fermions do not even induce a kinetic term for the gauge fields. We will therefore shift our focus away from the fermionic formulation (7) and concentrate on (2) more directly.

A different way in which (2) can arise as an effective field theory is from a renormalization group flow of an Abelian Higgs system

$$
L=\int d^{4} x N\left[-\frac{1}{4} F_{\mu \nu} F^{\mu \nu}-V\left(\frac{1}{\phi_{0}^{2}} D_{\mu} \phi^{*} D^{\mu} \phi\right)+V_{\phi}\left(\phi^{*} \phi\right)\right]
$$

where the Higgs potential $V_{\phi}$ is minimized at $|\phi|=\phi_{0}$, and the covariant derivative

$$
D_{\mu} \phi=\left(\partial_{\mu}+i A_{\mu}\right) \phi
$$

The standard Higgs mechanism will give rise to a massive scalar Higgs and three components of the vector field. The effective action (2) emerges by integrating out the massive Higgs. If the potential $V\left(A^{2}\right)$ has the form given in (3) , we see that this gives rise to a term in the effective action of the form

$$
L=-\frac{1}{\phi_{0}^{4}}\left(D_{\mu} \phi^{*} D^{\mu} \phi\right)^{2}
$$

with a negative coefficient. The criteria of [5] applies in classifying (14) as not having a consistent UV completion.

While these results are suggestive of (2) being inconsistent, one can still argue that it is a symptom specific to the massive Higgs field in (14) and not to the effective dynamics of (2). There should exist a separate argument showing that (2) itself, which can also be viewed as a gauged $U(1)$ sigma model in the unitary gauge, suffers from consistency issues of [5]. We will now provide arguments showing that this is indeed the case.

Consider specifically a model of the form which follows from (2) and (3)

$$
L=\int d^{4} x N\left[-\frac{1}{4} F_{\mu \nu} F^{\mu \nu}-\frac{\lambda}{4}\left(A_{\mu} A^{\mu}-v\right)^{2}\right], \quad \lambda>0, \quad v<0 .
$$

We will view this model as gauged $U(1)$ sigma model

$$
L=\int d^{4} x N\left[-\frac{1}{4} F_{\mu \nu} F^{\mu \nu}-\frac{\lambda}{4}\left(\left(\partial_{\mu} \sigma+A_{\mu}\right)\left(\partial^{\mu} \sigma+A^{\mu}\right)-v\right)^{2}\right], \quad \lambda>0, \quad v<0 .
$$

In this formulation, it is manifest that (17) is the result of the $\sigma$ field being eaten by the gauge fields. The dynamics of this Goldstone boson before coupling to the gauge field has 
the form

$$
N \lambda\left(\frac{v}{2} \partial_{\mu} \sigma \partial^{\mu} \sigma-\frac{1}{4}\left(\partial_{\mu} \sigma \partial^{\mu} \sigma\right)^{2}\right), \quad \lambda>0, \quad v<0 .
$$

Because $v$ is taken to be negative, this is precisely a model of the Ghost condensation type [23]. The $v$ and $\lambda$ are chosen so that $\partial \sigma$ is unstable to acquiring a space-like expectation value. Let us chose the vacuum so that

$$
\sigma=c x_{3}+\tilde{\sigma}
$$

As was explained in [23], there is a special value $c=c^{*}=\sqrt{-v}$ which the model will flow to under Hubble friction, but when decoupled from gravity, any $c>c^{*}$ will give rise to a stable vacuum. The effective action will take the form

$$
\begin{aligned}
L= & N \lambda\left[-c\left(c^{2}+v\right) \partial_{3} \tilde{\sigma}+\frac{\left(c^{2}+v\right)}{2} \partial_{i} \tilde{\sigma} \partial^{i} \tilde{\sigma}-\frac{\left(3 c^{2}+v\right)}{2}\left(\partial_{3} \tilde{\sigma}\right)^{2}+c \partial_{3} \tilde{\sigma}\left(\partial_{i} \tilde{\sigma} \partial^{i} \tilde{\sigma}-\left(\partial_{3} \tilde{\sigma}\right)^{2}\right)\right. \\
& \left.-\frac{1}{4}\left(\partial_{i} \tilde{\sigma} \partial^{i} \tilde{\sigma}-\left(\partial_{3} \tilde{\sigma}\right)^{2}\right)^{2}\right]
\end{aligned}
$$

where $i=0,1,2$. The term which is first order in $\tilde{\sigma}$ is a total derivative and can be ignored for this model. It is clear that when one restricts to the sector where $\partial_{3} \tilde{\sigma}=0$, this model is precisely the prototype (11) exhibiting the obstruction to UV completion. This is already a strong hint that (17) will exhibit similar pathology.

The remaining task is to show what happens to the dynamics of the $\tilde{\sigma}$ field when it is eaten by the $U(1)$ gauge field. If one chooses unitary gauge, this essentially amounts to replacing $\partial_{\mu} \tilde{\sigma}$ by $A_{\mu}$ in (21). This time, the linear term can not be ignored, and we are forced to set $c=c^{*}=\sqrt{-v}$. With this choice, the effective action of the form

$$
\begin{aligned}
L= & N \lambda\left[-\frac{1}{4 \lambda} F_{\mu \nu} F^{\mu \nu}+v A_{3}^{2}\right. \\
& \left.+\sqrt{-v} A_{3}\left(A_{i} A^{i}-A_{3}^{2}\right)-\frac{1}{4}\left(A_{i} A^{i}-A_{3}^{2}\right)^{2}\right]
\end{aligned}
$$

This effective action is manifestly invariant under the $2+1$ dimensional Lorentz symmetry.

In order to identify the manifestation of the UV obstruction, let us consider the forward scattering of $A_{i}$ in the $2+1$ Lorentz invariant subspace, but with a small amount of KaluzaKlein mass $\mu^{2}$ from momentum along the $x_{3}$ direction. Let us take $\mu^{2} \ll-\lambda v$ so that we can ignore the dynamics of the $A_{3}$ component. The effective action can then be written in the form

$$
L=N\left[-\frac{1}{4} F_{i j} F^{i j}+\frac{\mu^{2}}{2} A_{i} A^{i}-\frac{\lambda}{4}\left(A_{i} A^{i}\right)^{2}\right] .
$$


Now, let us chose to consider the forward scattering of the longitudinal modes for a collision along the $x_{1}$ axis. The momentum and the polarization of the ingoing particles will be set to

$$
p_{i}=(E, p, 0), \quad \epsilon_{i}=\frac{(p, E, 0)}{\mu}
$$

and

$$
p_{i}=(E,-p, 0), \quad \epsilon_{i}=\frac{(-p, E, 0)}{\mu}
$$

with $E^{2}-p^{2}=\mu^{2}$. The forward scattering amplitude due to the $\lambda\left(A_{i} A^{i}\right)^{2}$ term will evaluate in this case to

$$
\mathcal{M}=-\frac{\lambda}{N} \frac{s^{2}}{\mu^{4}}+\mathcal{O}(s) .
$$

We see that the $s^{2}$ term enters with a negative coefficient. This is precisely the signature of the obstruction to UV completion described in [5].

The main point of this note, in a nutshell, can be reduced to the arguments outlined between equations (17) and (26). Since the conclusion relies only on the sign of (26) which can be delicate to compute, we chose to include preliminary arguments leading up to this conclusion. Note, in particular, that the signs of (16) and (26) has the same origin.

It should be noted that the issues of UV completability have more to do with the sign of $v_{4}$ rather than the sign of $v_{2}$. Recall, in the discussion below (12), that the sign of $v_{2}$ dictated whether or not one expects $A_{\mu}$ to develop a vacuum expectation value. To keep the action properly bounded, however, it was necessary to keep $v_{4}$ positive. This conclusion is more general than the specific form of the potential (3) that we considered. Any generic potential

$$
V\left(A_{\mu} A^{\mu}\right)=V\left(A_{i} A^{i}-A_{3}^{2}\right)
$$

which is minimized at $A_{i}=0, A_{3}=\sqrt{-v}$ so that

$$
V^{\prime}(v)=0, \quad V^{\prime \prime}(v)>0
$$

will contain a term

$$
\frac{1}{2} V^{\prime \prime}(v)\left(A_{i} A^{i}\right)^{2}+\mathcal{O}\left(A_{3}\right)
$$

which enters the Lagrangian with a negative sign.

It might be worth pointing out that in terms of the original variable field $A_{\mu}$, the operator $v_{4}\left(A_{\mu} A^{\mu}\right)^{2}$ whose coefficient we identified as the diagnostic of the UV consistency following [5] is non-derivative unlike for the case of the scalar field in the example of (1). This is not too surprising in light of the fact that higher spin fields are in a certain sense more non-local than the spinless fields. 
In fact, it is also very unnatural for $v_{2}$ to take on a positive value since it amounts to assigning a wrong sign for the kinetic term of the Higgs field in (14). From the fermion model point of view, this corresponds to $\lambda_{2}$ being positive, which is also unnatural. Specifically, it can not arise from the exchange of massive vector bosons. The $\lambda_{2}$ coupling is indeed bounded in the case of the Thirring model. Although we did not find it in this article, it seems very natural for such bound to follow from the consideration of UV completability along the lines of [5] as well. This will provide additional perspective on the dynamical treatment of the fermion models [10, 12, 13, 16].

There are other examples of manifestly Lorentz invariant effective field theories which supports superluminal backgrounds in non-trivial backgrounds. One is the effective field theory of the " $k$-essence" type [24] whose Lagrangian takes the form

$$
L=\int d^{4} x f\left(\partial_{\mu} \phi \partial^{\mu} \phi\right)
$$

for some function $f 45$ Such an effective action have long been known to support superluminal propagation if $c_{s}^{2}=\left(1+2 X f^{\prime \prime} / f^{\prime}\right)^{-1}>1$ [26, 27, 28]. This is precisely the case for which $k$-essence models could have interesting cosmological implications [29]. Another example is a model of the form

$$
L=-\beta_{1} \partial^{\mu} A^{\sigma} \partial_{\mu} A_{\sigma}-\beta_{2}\left(\partial_{\mu} A^{\mu}\right)^{2}-\beta_{3} \partial^{\mu} A^{\sigma} \partial_{\sigma} A_{\mu}+\lambda\left(A^{\mu} A_{\mu}-m^{2}\right)
$$

which was considered in $[30,31,32,33,34,35]$. Here, $\lambda$ plays the role of the Lagrange multiplier constraining $A^{\mu} A_{\mu}=m^{2}$, where the case of $m^{2}>0$ corresponding to time-like (therefore distinct from the space-like condensate considered in [15]) vacuum expectation value was considered in [32,33], where it was shown that there will be superluminal propagating mode if $\left(\beta_{1}+\beta_{2}+\beta_{3}\right) / \beta_{1}>1$. These effective field theory models are excellent candidates to look for concrete signatures obstructing consistent UV completions, which we hope to identify in a future work. A non-trivial part of this program might involve systematic generalizations of the analysis of [5].

We should comment before closing that the obstructions to UV completion discussed in this note do not necessarily imply that such effective field theories are not realized in nature. The diagnostic of [5] is mainly the probe of analyticity. But nature may well be nonanalytic. It is still very interesting to study exotic models and unconventional frameworks in the search for possible solution to outstanding puzzles in effective field theory such as

\footnotetext{
${ }^{4}$ A special limit where $f(X)=\sqrt{X}$, and where the speed of sound is infinite, is the "cuscuton" model of 25$]$.

${ }^{5}$ Another possible pathology with the action of this form is that the map between velocity and momentum field variables may not be one to one, for example, if $f(X)=-a X+b X^{2}$ for $a, b>0$.
} 
the hierarchy problem and dark matter/dark energy. We just need to be aware that some models may require sacrificing of analyticity.

Let us also note that there is one context in which gauge bosons naturally arose as a consequence of spontaneous Lorentz symmetry breaking. Embedding of flat D3-brane in type IIB string theory gives rise to 6 massless scalar fields parameterizing the transverse embedding of the brane. These are Goldstone bosons. For the D3-branes in type IIB theory, however, there are enough supersymmetry to imply a presence of massless spin particle in its multiplet. There may well exist other, more elegant, ways to realize gauge fields as Goldstone particles in spontaneous breaking of Lorentz symmetry.

\section{Acknowledgements}

We would like to thank Daniel Chung and Ian Ellwood for discussions and collaboration at early stage of this work. We also thank Per Kraus for extensive correspondences, and Alexander Vikman for comments. We also thank Allan Adams, Baha Balenteken, Jaques Distler, Charlie Goebel, Tao Han, and Frank Petriello for discussions. This work was supported in part by the DOE grant DE-FG02-95ER40896 and funds from the University of Wisconsin.

\section{References}

[1] P. H. Frampton and C. Vafa, "Conformal approach to particle phenomenology," hep-th/9903226.

[2] J. Polchinski, "Effective field theory and the Fermi surface," hep-th/9210046.

[3] D. B. Kaplan, "Effective field theories," nucl-th/9506035.

[4] A. V. Manohar, "Effective field theories," hep-ph/9606222.

[5] A. Adams, N. Arkani-Hamed, S. Dubovsky, A. Nicolis, and R. Rattazzi, "Causality, analyticity and an IR obstruction to UV completion," JHEP 10 (2006) 014, hep-th/0602178.

[6] G. R. Dvali, G. Gabadadze, and M. Porrati, "4D gravity on a brane in 5D Minkowski space," Phys. Lett. B485 (2000) 208-214, hep-th/0005016.

[7] V. A. Kostelecky and S. Samuel, "Gravitational phenomenology in higher dimensional theories and strings," Phys. Rev. D40 (1989) 1886-1903. 
[8] R. Bluhm and V. A. Kostelecky, "Spontaneous Lorentz violation, Nambu-Goldstone modes, and gravity," Phys. Rev. D71 (2005) 065008, hep-th/0412320.

[9] R. Bluhm, S.-H. Fung, and V. A. Kostelecky, "Spontaneous Lorentz and diffeomorphism violation, massive modes, and gravity," arXiv:0712.4119 [hep-th].

[10] J. D. Bjorken, "A dynamical origin for the electromagnetic field," Annals Phys. 24 (1963) 174-187.

[11] Y. Nambu and G. Jona-Lasinio, "Dynamical model of elementary particles based on an analogy with superconductivity. I," Phys. Rev. 122 (1961) 345-358.

[12] T. Banks and A. Zaks, "Composite gauge bosons in four Fermi theories," Nucl. Phys. B184 (1981) 303.

[13] A. Jenkins, "Spontaneous breaking of Lorentz invariance," Phys. Rev. D69 (2004) 105007, hep-th/0311127.

[14] J. C. Collins, Renormalization. Cambridge University Press.

[15] P. Kraus and E. T. Tomboulis, "Photons and gravitons as Goldstone bosons, and the cosmological constant," Phys. Rev. D66 (2002) 045015, hep-th/0203221.

[16] J. Bjorken, "Emergent gauge bosons," hep-th/0111196.

[17] E. Abdalla, M. C. B. Abdalla, and K. D. Rothe, Nonperturbative methods in two-dimensional quantum field theory. World Scientific, Singapore.

[18] Y. Frishman and J. Sonnenschein, "Bosonization and QCD in two-dimensions," Phys. Rept. 223 (1993) 309-348, hep-th/9207017.

[19] M. Stone, Bosonization. World Scientific, Singapore.

[20] S. R. Coleman, "Quantum sine-Gordon equation as the massive Thirring model," Phys. Rev. D11 (1975) 2088.

[21] S. Mandelstam, "Soliton operators for the quantized sine-Gordon equation," Phys. Rev. D11 (1975) 3026.

[22] C. M. Naon, "Abelian and nonabelian bosonization in the path integral framework," Phys. Rev. D31 (1985) 2035.

[23] N. Arkani-Hamed, H.-C. Cheng, M. A. Luty, and S. Mukohyama, "Ghost condensation and a consistent infrared modification of gravity," JHEP 05 (2004) 074, hep-th/0312099. 
[24] C. Armendariz-Picon, V. F. Mukhanov, and P. J. Steinhardt, "Essentials of k-essence," Phys. Rev. D63 (2001) 103510, astro-ph/0006373.

[25] N. Afshordi, D. J. H. Chung, and G. Geshnizjani, "Cuscuton: A causal field theory with an infinite speed of sound," Phys. Rev. D75 (2007) 083513, hep-th/0609150.

[26] Y. Aharonov, A. Komar, and L. Susskind, "Superluminal behavior, causality, and instability," Phys. Rev. 182 (1969) 1400-1403.

[27] J. Garriga and V. F. Mukhanov, "Perturbations in k-inflation," Phys. Lett. B458 (1999) 219-225, hep-th/9904176.

[28] E. Babichev, V. Mukhanov, and A. Vikman, " $k$-Essence, superluminal propagation, causality and emergent geometry," arXiv:0708.0561 [hep-th].

[29] C. Bonvin, C. Caprini, and R. Durrer, "A no-go theorem for k-essence dark energy," Phys. Rev. Lett. 97 (2006) 081303, astro-ph/0606584.

[30] T. Jacobson and D. Mattingly, "Gravity with a dynamical preferred frame," Phys. Rev. D64 (2001) 024028, gr-qc/0007031.

[31] T. Jacobson and D. Mattingly, "Einstein-aether waves," Phys. Rev. D70 (2004) 024003, gr-qc/0402005.

[32] S. M. Carroll and E. A. Lim, "Lorentz-violating vector fields slow the universe down," Phys. Rev. D70 (2004) 123525, hep-th/0407149.

[33] E. A. Lim, "Can we see Lorentz-violating vector fields in the CMB?," Phys. Rev. D71 (2005) 063504, astro-ph/0407437.

[34] B. Li, D. Fonseca Mota, and J. D. Barrow, "Detecting a Lorentz-violating field in cosmology," arXiv:0709.4581 [astro-ph].

[35] T. Jacobson, "Einstein-aether gravity: a status report," arXiv:0801.1547 [gr-qc]. 